LA-UR-02-5375

Approved for public release; distribution is unlimited.

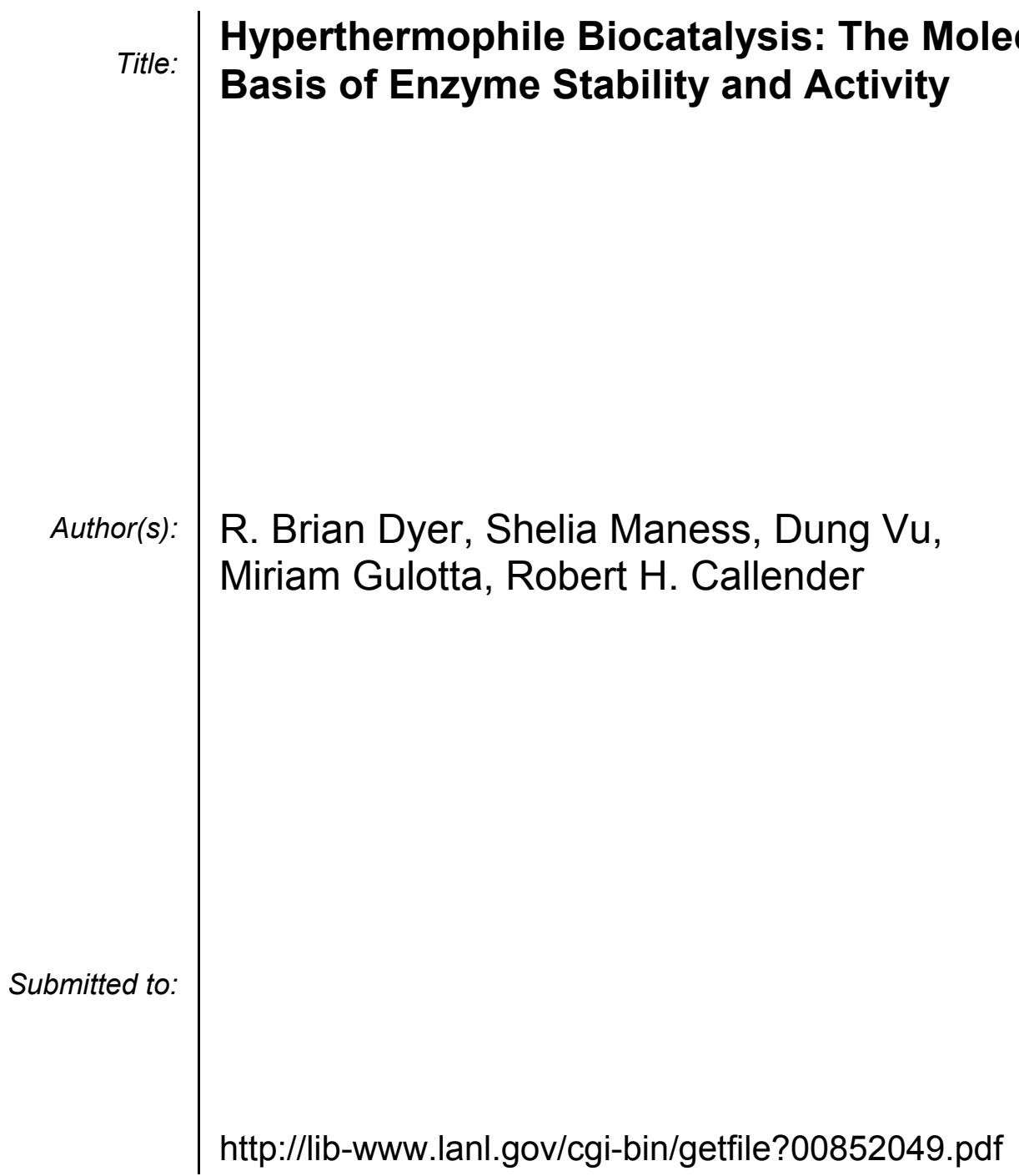

http://lib-www.lanl.gov/cgi-bin/getfile?00852049.pdf 


\title{
Hyperthermophile Biocatalysis: The Molecular Basis of Enzyme Stability and Activity
}

\author{
R. Brian Dyer*, Shelia Maness, Dung Vu, Miriam Gulotta, Robert H. Callender (Albert Einstein \\ College of Medicine)
}

\begin{abstract}
The motions of key residues at the substrate binding site of lactate dehydrogenase (LDH) from thermophilic and mesophilic organisms were probed using laser induced temperature jump relaxation spectroscopy employing both UV fluorescence and isotope edited IR absorption spectroscopy as structural probes. The dynamics associated with loop closure are observed to involve several steps with motions from 1-300 $\mu \mathrm{s}$. Apart from the 'melting' of a few residues on the protein's surface, no kinetics were observed on any time scale in experiments of the bound NAD-pyr adduct, even for final temperatures close to the unfolding transition. This is contrary to simple physical considerations and models. These results show that, once a productive protein/substrate complex is formed, the binding pocket is very rigid with very little, if any, motion apart from the mobile loop. The results provide fundamental new insight into the molecular mechanism of this important class of enzymes.
\end{abstract}

\section{Background and Research Objectives}

Lactate dehydrogenase (LDH) accelerates the reduction of pyruvate by NADH to lactate and $\mathrm{NAD}^{+}$by about $10^{14}$-fold relative to the corresponding model reaction (1). The reaction involves the direct transfer of a hydride ion from $\mathrm{C} 4$ of the reduced nicotinamide group of NADH to the $\mathrm{C} 2$ carbon of pyruvate (Figure 1). The nature of the LDH/NADH/pyruvate (ternary) complex has been studied using an adduct complex, E/NAD-pyr, that is formed by the addition of the $\mathrm{C} 3$ carbon of pyruvate enol to the $\mathrm{C} 4$ position of the nicotinamide ring of $\mathrm{NAD}^{+}$in the presence of $\mathrm{LDH}(1)$. The dissociation constant for the adduct is about $10^{-10} \mathrm{M}$, just slightly smaller (about a factor of ten) than the product of the dissociation constants for LDH with NADH and E/NADH with pyruvate. The pyruvate moiety of this adduct likely interacts with the same residues that interact with pyruvate in its ternary complex with LDH based on X-ray structural (2$5)$, chemical $(1,6,7)$, as well as Raman structural $(8,9)$ studies. Oxamate $\left(\mathrm{NH}_{2} \mathrm{COCOO}^{-}\right)$is an inhibitor of LDH and is an isoelectronic and isosteric mimic of pyruvate. It dissociation constant is smaller than that of pyruvate $\left(\mathrm{K}_{\mathrm{d}}\right.$ of $20 \mu \mathrm{M}$ compared to $\left.5 \mathrm{mM}\right)$. However, it too binds in a structural arrangement believed to mimic pyruvate binding. Moreover, it has been shown to have very similar binding kinetics to that of pyruvate $(10,11)$.

While the dynamics of the catalytic reaction are unknown, we postulate the following crude outline of the initial dynamics of enzyme catalyzed conversion of pyruvate to lactate-. Pyruvate forms some sort of encounter complex with LDH/NADH. Once the substrate reaches positioning close enough to the enzyme's active site, which is buried into the enzyme, a loop of the polypeptide chain, residues 98-110, closes over the active site entrance, water leaves the binding pocket, the enzyme tightens around the bound substrate, and key residues are brought in close proximity to the substrate. At this point, it is probable that the structural arrangement is close to what is formed in ternary complexes observed in the crystallographic studies. 
Figure 1 shows a cartoon of $\mathrm{NADH}$ and pyruvate and its arrangement with specific active site residues of $\mathrm{LDH}$. There are several charged residues that are important for catalysis and binding. The His-195 and Asp-168 dyad are essential for catalysis. N3 of the protonated imidazole ring of His-195 approaches the carbonyl ring oxygen of pyruvate at an angle that is optimal for hydride transfer which must occur in the pyruvate to lactate transformation. In addition, a strong hydrogen bond is formed between the His-195 and the substrate carbonyl oxygen that polarizes the $\mathrm{C}=\mathrm{O}$ bond. The loop closure of residues 98-110 brings Arg-109 also close to the substrate carbonyl oxygen forming another hydrogen bond. It is known that electrostatic stabilization of transition state in the pyruvate-lactate interconversion, which contains a highly polarized carbonyl moiety, ${ }^{+} \mathrm{C}_{-} \mathrm{O}^{-}$, is responsible for about half of the rate enhancement brought about by LDH $(1,9)$. The enzyme induced carbonyl bond polarization shows up as a downward frequency shift in the $\mathrm{C}=\mathrm{O}$ stretch mode (located at $1710 \mathrm{~cm}^{-1}$ in solution) of $35 \mathrm{~cm}^{-1}$ (9). It has been determined that the His-195/Asp-168 dyad is responsible for about $24 \mathrm{~cm}^{-1}$ of this frequency shift and Arg-109 for about $11 \mathrm{~cm}^{-1}$. Another key residue is Arg-171, which solvates the ionized carboxylic moiety of pyruvate at the binding site; this interaction affects the stretch modes of the ionized carboxyl moiety raising its antisymmetric stretch frequency by $20 \mathrm{~cm}^{-1}$ (1615 to 1635 $\mathrm{cm}^{-1}$, see below).

We use the NAD-pyr adduct as a probe of the dynamics taking place at the active site. This molecule does not undergo catalysis, and it does not dissociate from LDH. It is different from the normal ternary productive complex in that the NADH cofactor and the pyruvate substrate are tied together through the covalent bond between the $\mathrm{C} 4$ position of $\mathrm{NADH}$ and the $\mathrm{C} 2$ carbon of pyruvate. Hence, any relative motion between these normally reacting species is frozen out. This simplifies the system to a point where several important questions concerning specific dynamical features of the active site can be answered.

The central issue we pose in this study is, once pyruvate is bound to $\mathrm{LDH}$, what are the relative motions of the key residues in the protein binding pocket with respect to the bound substrate. The dynamics of loop opening/closing are of particular interest. Holbrook and his colleagues $(10,11)$, in kinetic studies of substrate and inhibitor binding to LDH/NADH, have shown that the loop opening rate is $580 \mathrm{~s}^{-1}$ and loop closure occurs at $3020 \mathrm{~s}^{-1}$ at $23{ }^{\circ} \mathrm{C}$. We have confirmed these rates in T-jump relaxation experiments of the LDH/NADH complex when it binds oxamate using the emission properties of the nicotinamide headgroup of NADH as a marker of loop open/closing. Our studies also show two faster processes, showing that loop closure involves several steps. Loop motion and its affect on bound substrate can be monitored as well through shifts in frequency of the $\mathrm{C}=\mathrm{O}$ stretch of the bound NAD-pyr adduct. The breaking and/or weakening of the hydrogen bond between Arg-171 and substrate yields up to the $11 \mathrm{~cm}^{-1}$ frequency shift in this $\mathrm{C}=\mathrm{O}$ stretch. In this study, we have found it possible to determine a shift in this bond stretching frequency of as little as one $\mathrm{cm}^{-1}$ and smaller over time scales of $20 \mathrm{~ns}$ to 20 ms using our IR T-jump relaxation spectrometer. Likewise, any relative motion between the His-195/Asp-168 dyad and substrate $\mathrm{C}=\mathrm{O}$ moiety shows up as a substantial shift in the substrate $\mathrm{C}=\mathrm{O}$ stretch frequency. Motion against the substrate's carboxyl group can be assessed by shifts in the antisymmetric stretch frequency of a $-\mathrm{COO}^{-}$moiety which lies at $1616 \mathrm{~cm}^{-1}$ in solution. The frequency of this band is upshifted by the formation of hydrogen bonds and salt bridges and so the dynamics of the making/breaking of these interactions show up as frequency shifts of this internal coordinate. In this way, the relative dynamics of the substrate against its protein host can be characterized quite thoroughly and specifically.

\section{Importance to LANL's Science and Technology Base and National R\&D Needs}

We have developed a unique capability at LANL to study protein dynamics that determine how proteins fold and then perform their essential functions. Understanding protein dynamics is thereby a crucial component of understanding the data generated by the human genome project, and other sequencing efforts. Our picture of the molecular nature of enzymatic catalysis typically 
revolves around static structures of enzymes, with and without bound substrates and inhibitors, as determined from crystallographic, multiple dimension NMR, or vibrational spectroscopy . This is necessarily a limited view, providing only 'snapshots' of the development of the reaction coordinate and providing no sense of the timing of various events. Indeed, the theory of transition state stabilization, invoked to explain the observed rate enhancements produced by enzymes over the corresponding chemical reactions in solution, is also a static picture. Modern paradigms for enzymatic catalysis can and do include atomic motion; however, little experimental data support these theories. A basic reason for the paucity of an experimental understanding has to do with technical limitations. It has, until recently, been very difficult to observe atomic motion on the $10 \mathrm{~ns}$ to $1 \mathrm{~ms}$ time scales. We have developed a unique capability at LANL that applies laser induced temperature jump relaxation spectroscopy to study aspects of the dynamics occurring in the active sites of enzymes. Laser light tuned to the near IR is used to irradiate weak water bands. The subsequent thermalization of the excited water molecules results in a prompt temperature increase, as fast as picoseconds. Temperature jumps can provide the 'trigger' initiating a reaction or conformational change, and the conformational change can be monitored by a number of spectroscopies. We have also developed time resolved IR and fluorescence spectroscopies as probes of the protein dynamics with high structural and temporal resolution.

\section{Scientific Approach and Accomplishments}

T-jump relaxation spectroscopy perturbs the equilibrium point of inter-converting chemical species by rapidly changing the temperature, forcing the system to establish a new equilibrium point. The spectrometers used here heat water by pumping weak water absorption bands in the near IR and employ fluorescence and mid IR as probes of structure. For these systems, the time evolution of reaching the new equilibrium point is determined with a resolution of about $15 \mathrm{~ns}$. The technique relies on the existence of an enthalpic difference between the old and new equilibrium points. It can be shown (21) that the change in equilibrium between two inter-converting species for a given jump temperature is given by:

$$
\frac{\Delta K}{K}=5.67 \cdot 10^{-3} \Delta H \cdot \Delta T
$$

where $\mathrm{K}$ is the equilibrium constant, $\Delta \mathrm{H}$ is the enthalpy difference between the two species (in $\mathrm{kcal} / \mathrm{mol}$ ), and $\Delta \mathrm{T}$ is the temperature jump (in degrees Kelvin). Our average T-jump is $15^{\circ} \mathrm{C}$ and our sensitivity to changes in $\mathrm{K}$ in the present experiments is approximately $\Delta \mathrm{K} / \mathrm{K}=0.04$ or better. This implies that any two states separated by as little as $0.5 \mathrm{kcal} / \mathrm{mol}$ in enthalpy will be observed. The polar interactions at the binding site of LDH between protein and substrate involve substantially larger enthalpic interactions than this, as discussed below. For example, the binding enthalpy of pyruvate or the inhibitor oxamate to $\mathrm{LDH} / \mathrm{NADH}$ is $15-18 \mathrm{kcal} / \mathrm{mol}$ (22). Hence, $\mathrm{LDH}$ is an appropriate system for T-jump relaxation spectroscopy in an examination of its catalytic dynamics.

We begin this discussion by asking what type of dynamics we might expect to occur. In general, we might expect any protein complex to adopt a range of conformational substates, with these substates possessing a range of functional efficacy. For example, it has been observed that the binding of NADH to LDH is a multistep process, with dynamics observed on multiple time scales from nanoseconds to milliseconds (17). It was found that the LDH/NADH complex does not adopt a single structure. The binary complex adopts multiple interconverting structures of significant population; some of these structures appear to be far from catalytically productive. Hence, one important question in the present study is whether or not the ternary protein/cofactor/substrate complex also consists of multiple interconverting structures and, if so, are some of these either catalytically non-productive or less productive compared to others. This possibility is reinforced by our recent observation (unpublished data) that multiple vibrational bands indicating multiple populated conformations are observed for the catalytically key $\mathrm{C} 4-\mathrm{H}$ stretch coordinate of NADH bound as the LDH/NADH/oxamate complex, with theoretical considerations 
suggesting that only one of these species can be close to the productive geometry. Moreover, the $\mathrm{C}=\mathrm{O}$ stretch mode of bound pyruvate is quite broad (see Results), some of which may be the result of a heterogeneous broadening mechanism brought about by a distribution of interactions at the binding site. Clearly heterogeneously broadened bands for the $\mathrm{C}=\mathrm{O}$ stretch of bound pyruvate when bound to mutants of $\mathrm{LDH}$ have been observed (9). Since the $\mathrm{C}=\mathrm{O}$ stretch frequency is a direct monitor of $\mathrm{k}_{\mathrm{cat}}$, with a $34 \mathrm{~cm}^{-1}$ shift corresponding to a factor of $10^{5.5}$ rate enhancement of the hydride transfer (9), a particular substrate's catalytic efficiency can be assessed if the frequency distribution of the heterogeneous ensemble is determined.

The known characteristic rates of loop closure/opening when substrate binds to the LDH/NADH binary complex are $3600 \mathrm{~s}^{-1}$ at $23{ }^{\circ} \mathrm{C}(11)$ and $8400 \mathrm{~s}^{-1}$ at $41{ }^{\circ} \mathrm{C}$ (see Results). The structural event that is being monitored on these time scales is one that quenches the fluorescence quantum yield of the reduced nicotinamide ring as the ring closes over the binding site. This is probably the last event, or very close to the last event, in the process of loop closure since it is well known that the emission of NADH in the 'closed' ternary LDH/NADH/substrate ternary complex is almost completely quenched. Loop closure is of substantial importance to the chemistry of catalysis in LDH; it enhances $\mathrm{k}_{\text {cat }}$ in LDH by over a factor 1200 (23). For a simple model of the dynamics that involves the loop closing over the substrate binding site with substrate more or less in place, the kinetics of loop closure should be observed in experiments on the LDH/NAD-pyr adduct complex since the frequency of the adduct's $\mathrm{C}=\mathrm{O}$ stretch shifts substantially upward upon loop opening (9).

Finally, the local energies of the hydrogen bonds between various groups of LDH and the $\mathrm{C}=\mathrm{O}$ group of the pyruvate substrate have been deduced from their effect of polarizing the $\mathrm{C}=\mathrm{O}$ bond through shifts in the $\mathrm{C}=\mathrm{O}$ stretch mode for bound pyruvate (9). The values are 11 and 4 $\mathrm{kcal} / \mathrm{mol}$ between the $\mathrm{C}=\mathrm{O}$ group and the key active site His-195/Asp-168 dyad and the loop containing Arg-109, respectively. The breaking of the $\mathrm{C}=\mathrm{O} \bullet \bullet A r g-109 \mathrm{H}$-bond is clearly not too difficult and may be expected to break and form many times during the ca. $1 \mathrm{~ms}$ time that it takes for catalytic chemistry to occur in LDH. The breaking of the $\mathrm{C}=\mathrm{O} \bullet \bullet H i s-195$ bond, although substantial, may well also occur. For example, using a Boltzman distribution that describes the thermal population of states and a pre-exponential factor of $10^{13} \mathrm{sec}^{-1}$, it is predicted that the $\mathrm{C}=\mathrm{O} \bullet \bullet$ His- 195 bond will break in about $10 \mu \mathrm{s}$. Interestingly, this time is on the order of the observed characteristic time of loop closure. ??why is this significant - are we implying that loop closure and the $\mathrm{C}=\mathrm{O} \bullet \bullet H$ is $\mathrm{H}$-bond formation are somehow linked??

In order to focus on the dynamics occurring at the binding site of $\mathrm{LDH}$ once substrate is bound, we have performed studies on the LDH/NAD-pyr complex. The actual process of forming an encounter complex in the binding of substrate to enzyme has already occurred so that these dynamics are 'frozen out'. Substrate binding dynamics are studied separately in experiments, for example, of the binding of the inhibitor oxamate to $\mathrm{LDH} / \mathrm{NADH}(10,11$; see Results).

Furthermore, the LDH/NAD-pyr adduct is unable to undergo hydride transfer so that the kinetics associated with the chemistry transforming substrate to product do not complicate the interpretations of the results. The LDH/NAD-pyr adduct is neither a strict mimic of the ground state Michaelis complex nor that of the transition state, but rather has features of both. The NAD cofactor is covalently bound to the pyruvate substrate. In this sense, the two molecules which would normally react at the active site, are kept close to each other. Such a close encounter complex between cofactor and substrate is believed to be fairly advanced towards the transition state along the reaction pathway, and its formation is required for catalysis (24). These 'nearest approach complexes' (so-called NACs) are probably formed and broken on the sub-ns time scale in LDH (25). On the other hand, the structural arrangement and interactions of the carboxyl and carbonyl groups of the pyruvate moiety of the NAD-pyr adduct with its protein environment when bound to $\mathrm{LDH}$ is believed to mimic quite well what would be encountered with a true Michaelis ground state LDH/NADH/pyruvate ternary complex (1-9).

The results on the LDH/NAD-pyr adduct complex shows no dynamical features from $20 \mathrm{~ns}$ to out to at least the millisecond time scale apart from the 'melting' of a few residues on the protein's 
surface and, hence, none that would affect catalysis over this time scale. The experiments are quite sensitive. Virtually any change in the distances between the pyruvate group's $\mathrm{C}=\mathrm{O}$ bond and - $\mathrm{COO}^{-}$group with regards the key active site residues His-195, Asp-168, Arg-109, and/or Arg-171 would have shown up as shifts in the $\mathrm{C}=\mathrm{O}$ or $-\mathrm{COO}^{-}$stretch bands. No shifts were observed with a sensitivity of around 30/1. It should be noted that our experiments reached a final temperatures near $50{ }^{\circ} \mathrm{C}$, which is close to the unfolding temperature of $\mathrm{LDH}\left(\mathrm{T}_{\mathrm{m}}=62{ }^{\circ} \mathrm{C}\right.$ in the rabbit muscle protein; 26). We conclude that the active site groups in $\mathrm{LDH}$ around the pyruvate moiety of the NAD-pyr adduct are quite immobilized and rigid. If there are populated protein substates which include a different geometric arrangement of the polar groups at LDH's active site, the enthalpy difference between them is less than $0.5 \mathrm{kcal} / \mathrm{mol}$ or less than the $0.6 \mathrm{kcal}$ thermal energy at room temperature. Given the strengths of the various hydrogen bonds at the active site and the sizable enthalpies involved in these bonds, there would appear virtually no distribution of substates that involve perturbations of these bonds. To the extent that the NAD-pyr complex resembles a NAC complex, and hence a bound structure advanced towards the transition state, it is concluded that the advancement towards the transition state involves a 'freezing out' of substate conformations.

A surprising result of this study is the lack of any kinetics associated with loop closure, in the LDH/NAD-pyr complex which was shown in Results to occur at $119 \mu \mathrm{s}$ (at $41^{\circ} \mathrm{C}$ ) in experiments of the binding of oxamate to LDH/NADH. A simple binding model would have the substrate enter into the active site with the mobile loop in an open conformation. Once the substrate orientates itself within the binding pocket, the loop closes over the substrate. In the reverse of this process, the mobile loop breaks contact with substrate in the LDH/NADH/pyruvate ternary complex, breaking among other interactions the H-bond between $\mathrm{C}=\mathrm{O} \bullet \bullet A \mathrm{Arg}-109$, followed by the leaving of the substrate. The present results show that this model can not describe the dynamics of loop motion given that no changes in the $\mathrm{C}=\mathrm{O}$ stretch mode were observed in the LDH/NAD-pyr complex. The formation of the adduct complex, which ties the pyruvate moiety to the binding site because it is covalently linked to bound NAD, clearly greatly slows loop opening (to a time where we can not observe it). It can be concluded from our Results that the simple model described above can not describe loop dynamics but rather points to loop opening motion that involves a coordinated motion of the substrate as well as the loop. It is clear that the substrate must move, either with or in some partial way, with the protein loop. The kinetics of product release from LDH, which is rate limiting in the enzyme catalyzed reaction, appear to rely on a small substrate and its concomitant mobility.

\section{Publications}

1. M. Gulotta, H. Deng, H. Deng, R. B. Dyer and R. H. Callender, "Towards and Understanding of the Role of Dynamics on Enzymatic Catalysis in Lactate Dehydrogenase," Biochemistry 41, 3353-3363, (2002).

2. S. Franzen, J. Bailey, R. B. Dyer, W. H. Woodruff, R. B. Hu, M. R. Thomas, S. G. Boxer, "A Photolysis-Triggered heme Ligand Switch in H93G Myoglobin," Biochemistry 40, 5299-5305 (2001).

3. R. B. Dyer, M. Gulotta, H. Deng, R. H. Callender, "The Functional Dynamics of Lactate Dehydrogenase: Loop Closure and Hydride Transfer," Biochemistry submitted, (2002).

\section{References.}

1. Burgner, J. W., \& Ray, W. J. Biochemistry 23, 3636-3648 (1984).

2. Holbrook, J. J., Liljas, A., Steindel, S. J., \& Rossmann, M. G. in The Enzymes (Boyer, P. D., Ed.) pp 191-293, Academic Press, New York (1975). 
3. White, J. L., Hackert, M. L., Buehner, M., Adams, M. J., Ford, G. C., Lentz, P. J., Smiley, I. E., Steindel, S. J., \& Rossmann, M. G. J. Mol. Biol. 102, 759-779 (1976).

4. Grau, U. M., Trommer, W. E., \& Rossmann, M. G. J. Mol. Biol. 151, 289-307 (1981).

5. Wigley, D. B., Gamblin, S. J., Turkenburg, J. P., Dodson, E. J., Piontek, K., Muirhead, H., \& Holbrook, J. J. J. Mol. Biol. 223, 317-335 (1992).

6. Burgner, J. W., \& Ray, W. J. Biochemistry 23, 3620-3626 (1984).

7. Burgner, J. W., \& Ray, W. J. Biochemistry 23, 3626-3635 (1984).

8. Deng, H., Zheng, J., Burgner, J., \& Callender, R. Proc. Nat'l. Acad. Sci. (USA) 86, 4484-4488 (1989).

9. Deng, H., Zheng, J., Clarke, A., Holbrook, J. J., Callender, R., \& Burgner, J. W. Biochemistry 33, 2297-2305 (1994).

\section{Figures.}

Figure 1. A diagram of the binding site of LDH with bound NADH and pyruvate showing hydrogen bonds between the substrate and key catalytically important residues of the protein. The catalytic event involves the hydride transfer of the $\mathrm{C} 4$ hydrogen of NADH from the pro-R side of the reduced nicotinamide ring to the $\mathrm{C} 2$ carbon of pyruvate and protein transfer from the imidazole group of His-195 to pyruvate's keto oxygen.

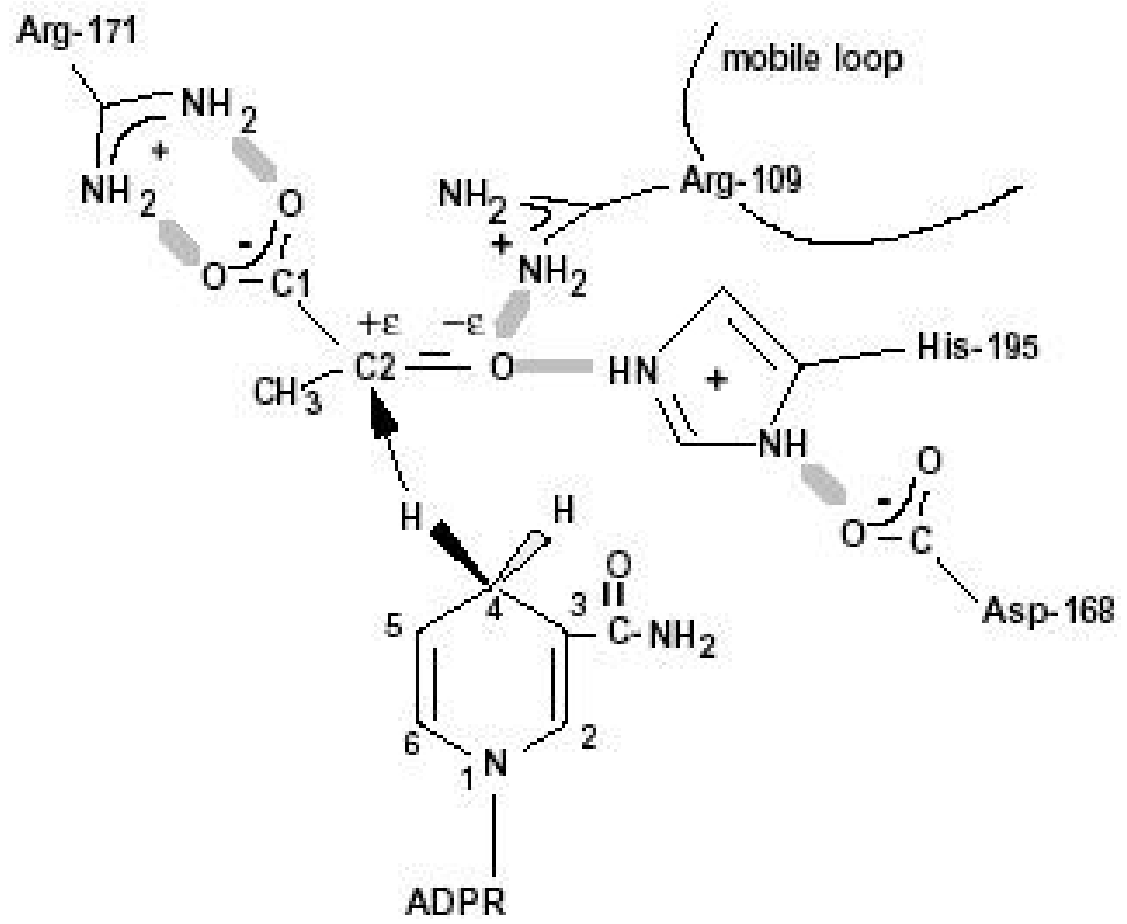


Figure 2. The kinetics of the Trp emission of apo-LDH measured at $340 \mathrm{~nm}$ in response to a Tjump from 10 to $26^{\circ} \mathrm{C}$. The early time kinetics were fitted to a three exponential function with time constants of 12 , and $17 \mathrm{~ns}$ and $3.5 \mu \mathrm{s}$, which is overlaid on the data. The emission kinetics show a slow recovery on the millisecond time scale from heat diffusion out of the laser interaction volume returning to the original temperature of $10^{\circ} \mathrm{C}$. The protein concentration was $100 \mu \mathrm{N}$ in $100 \mathrm{mM}$ sodium phosphate $\mathrm{pH}=7.2$.

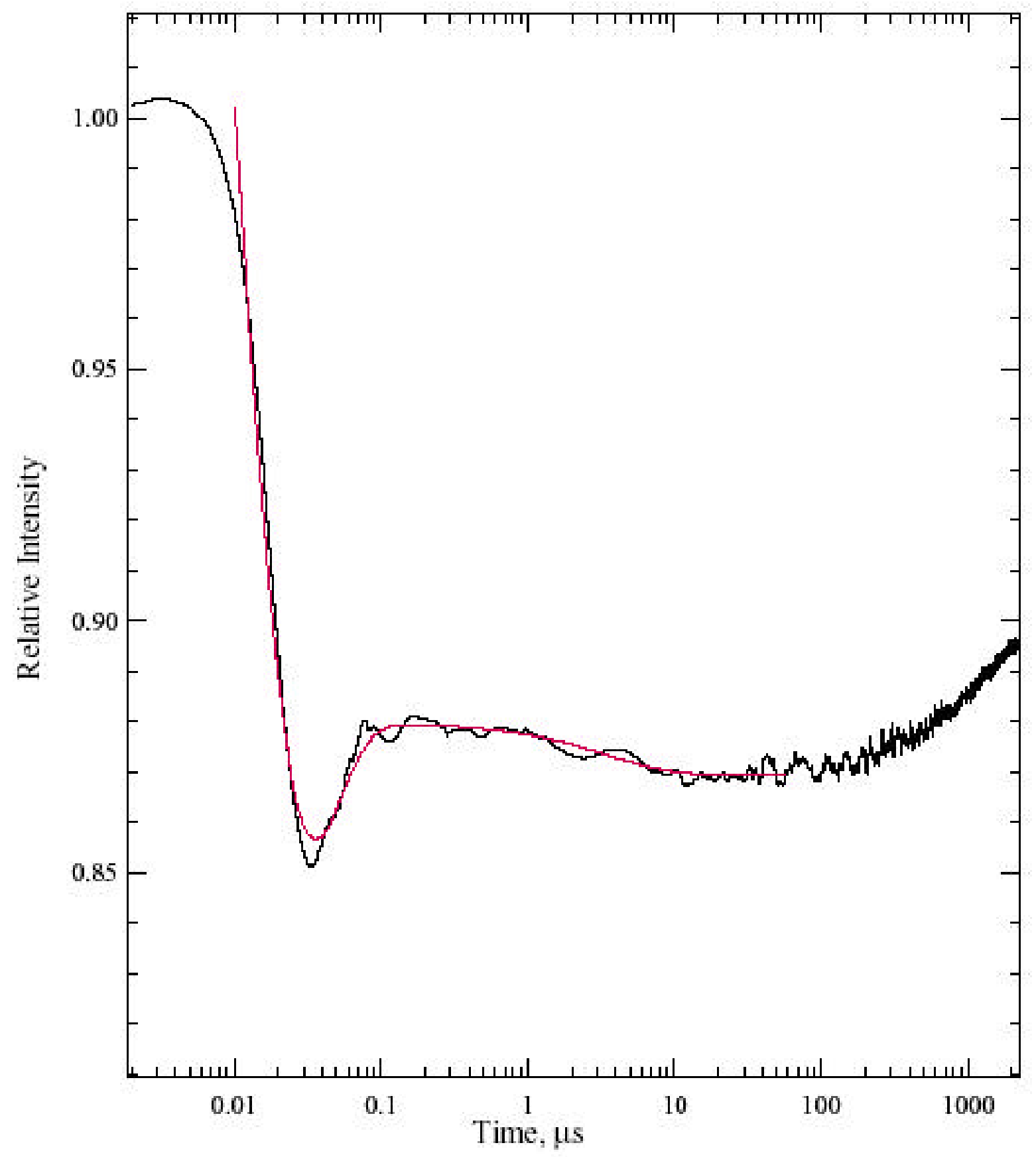

